\title{
N-Acetylcysteine Inhibits Muscle Fatigue in Humans
}

\author{
Michael B. Reid, Dobrivoje S. Stokić, Stephen M. Koch, Fadi A. Khawli, and A. Arturo Leis \\ Department of Medicine and Division of Restorative Neurology and Human Neurobiology, Baylor College of Medicine, Houston, Texas \\ 77030; Department of Anesthesiology, The University of Texas Medical School, Houston, Texas 77030; and Department of Neurology, \\ University of Mississippi Medical Center, Jackson, Mississippi 39216
}

\begin{abstract}
$\mathrm{N}$-acetylcysteine (NAC) is a nonspecific antioxidant that selectively inhibits acute fatigue of rodent skeletal muscle stimulated at low (but not high) tetanic frequencies and that decreases contractile function of unfatigued muscle in a dose-dependent manner. The present experiments test the hypothesis that NAC pretreatment can inhibit acute muscular fatigue in humans. Healthy volunteers were studied on two occasions each. Subjects were pretreated with NAC 150 $\mathrm{mg} / \mathrm{kg}$ or $5 \%$ dextrose in water by intravenous infusion. The subject then sat in a chair with surface electrodes positioned over the motor point of tibialis anterior, an ankle dorsiflexor of mixed-fiber composition. The muscle was stimulated to contract electrically $(40-55 \mathrm{~mA}, 0.2-\mathrm{ms}$ pulses) and force production was measured. Function of the unfatigued muscle was assessed by measuring the forces produced during maximal voluntary contractions (MVC) of ankle dorsiflexor muscle groups and during electrical stimulation of tibialis anterior at $1,10,20,40,80$, and $120 \mathrm{~Hz}$ (protocol 1). Fatigue was produced using repetitive tetanic stimulations at $10 \mathrm{~Hz}$ (protocol 1) or $40 \mathrm{~Hz}$ (protocol 2); intermittent stimulations subsequently were used to monitor recovery from fatigue. The contralateral leg then was studied using the same protocol.

Pretreatment with NAC did not alter the function of unfatigued muscle; MVC performance and the force-frequency relationship of tibialis anterior were unchanged. During fatiguing contractions stimulated at $10 \mathrm{~Hz}$, NAC increased force output by $\sim 15 \%(P<0.0001)$, an effect that was evident after 3 min of repetitive contraction $(P$ $<0.0125$ ) and persisted throughout the 30 -min protocol. NAC had no effect on fatigue induced using $40 \mathrm{~Hz}$ stimuli or on recovery from fatigue. $N$-acetylcysteine pretreatment can improve performance of human limb muscle during fatiguing exercise, suggesting that oxidative stress plays a causal role in the fatigue process and identifying antioxidant therapy as a novel intervention that may be useful clinically. (J. Clin. Invest. 1994. 94:2468-2474.) Key words: skeletal muscle - excitation-contraction coupling - fatigue $\bullet$ oxidative stress $\bullet$ free radicals
\end{abstract}

Address correspondence to Michael B. Reid, PhD, Pulmonary Medicine, Baylor College of Medicine, One Baylor Plaza, Suite 520 B, Houston, TX 77030. 1994.

Received for publication 9 May 1994 and in revised form 15 July

J. Clin. Invest.

(C) The American Society for Clinical Investigation, Inc.

$0021-9738 / 94 / 12 / 2468 / 07 \quad \$ 2.00$

Volume 94, December 1994, 2468-2474

\section{Introduction}

Acute muscular fatigue is associated with the development of oxidative stress in humans. Strenuous contraction increases free radical production by human limb muscle (1) and fatiguing exercise alters biochemical indices of oxidative stress including glutathione status $(2,3)$ and markers of lipid peroxidation $(4$, $5)$. Such biochemical changes can be inhibited by chronic supplementation with antioxidant vitamins $(4,5)$ but nutritional supplements have not improved exercise performance (6-9). Thus, experiments to date have not determined whether oxidative stress is a cause of muscular fatigue in humans or simply an effect.

In order to establish a cause/effect relationship, it is critical to demonstrate that exogenous antioxidants inhibit the loss of mechanical function during fatigue. This study tested this relationship using an experimental design that differs from previous human studies in two aspects. First, rather than using chronic nutritional supplements, we pretreated subjects with the nonspecific antioxidant $N$-acetylcysteine (NAC) ${ }^{1}(10)$ by intravenous infusion immediately before fatiguing exercise. Second, rather than using volitional exercise to induce peripheral fatigue, we electrically stimulated tibialis anterior to repetitively contract under quasi-isometric conditions. This technique was developed previously (11) to evoke peripheral fatigue in the absence of central neural processes (e.g., inhibitory reflexes, coordination, motivation, learning) that can importantly influence volitional performance. Data from two experimental protocols were used to test three hypotheses.

Hypothesis 1. $\mathrm{N}$-acetylcysteine inhibits fatigue of human tibialis anterior during low-frequency stimulation. Repetitive tetanic contractions produced using low stimulus frequencies cause mechanical losses and energetic changes that closely mimic muscular fatigue under physiologic conditions (12). $\mathrm{N}$ acetylcysteine inhibits fatigue of isolated muscle bundles during low-frequency stimulation in vitro $(13,14)$ and intravenous infusion of $N$-acetylcysteine $150 \mathrm{mg} / \mathrm{kg}$ inhibits fatigue of rabbit diaphragm in situ (15). We used similar methodology to test $\mathrm{N}$-acetylcysteine effects on fatigue of human limb muscle during low-frequency stimulation (protocol 1 ).

Hypothesis 2. Fatigue stimulated at high frequency and acute recovery from fatigue are unaffected by $\mathrm{N}$-acetylcysteine. Repetitive tetanic contractions produced using high stimulus frequencies causes rapid loss of mechanical function that has a different cellular mechanism than fatigue induced using low stimulus frequencies (12). Termination of either low- or highfrequency stimulation permits reversal of fatigue and recovery of mechanical function. A limited amount of data from animal experiments suggest that antioxidant administration may not

1. Abbreviations used in this paper: CNS, central nervous system; MVC, maximal voluntary contraction; $\mathrm{N}$, Newtons; NAC, $\mathrm{N}$-acetylcysteine. 
affect either fatigue induced using high stimulus frequencies $(16,17)$ or acute recovery from fatigue $(15,17)$ but such interventions have not been evaluated in humans. We therefore tested $\mathrm{N}$-acetylcysteine effects on fatigue at high stimulus frequencies (protocol 2) and on recovery from fatigue (protocols 1 and 2 ).

Hypothesis 3. Systemic administration of $N$-acetylcysteine $150 \mathrm{mg} / \mathrm{kg}$ does not alter the strength or contractile properties of unfatigued muscle. $N$-acetylcysteine depresses the contractile properties of unfatigued muscle studied in vitro (14). This effect is dose-dependent (14), however, and systemic administration of $\mathrm{N}$-acetylcysteine $150 \mathrm{mg} / \mathrm{kg}$ to anesthetized rabbits does not depress contractile function of unfatigued diaphragm (15). Accordingly, we hypothesized that the same dose of $\mathrm{N}$-acetylcysteine administered to humans would not decrease the strength or contractile properties of unfatigued limb muscle (protocol 1).

\section{Methods}

\section{Subjects}

We recruited 10 healthy men for this study; 3 participated in both protocols. On average they were $32 \mathrm{yr}$ old $( \pm 7 \mathrm{SD})$ and weighed 75 $\mathrm{kg}( \pm 12 \mathrm{SD})$. Each gave informed consent and was studied using procedures approved by the Baylor Institutional Review Board for Human Research. Subjects refrained from strenuous exercise for $48 \mathrm{~h}$ before each experiment. Right and left tibialis anterior muscles were tested in each of five subjects using protocol $1 ; 16$ muscles from eight subjects were tested using protocol 2.

\section{Drug administration}

The prevalence and severity of reactions to $\mathrm{N}$-acetylcysteine (see Results) made it impossible to blind investigators or subjects. In each protocol, therefore, a cross-over design was used. Subjects were studied on two occasions, one treatment trial and one control trial, such that each muscle served as its own control. Immediately before every trial, a physician investigator placed a 22-gauge catheter in the subject's forearm vein and $500 \mathrm{ml}$ of $5 \%$ dextrose in water was administered by infusion pump over $1 \mathrm{~h}$. For treatment trials, the infusion contained pyrogen-free $\mathrm{N}$-acetylcysteine (Mucomyst Intravenous; Apothecon, Princeton, NJ) $150 \mathrm{mg} / \mathrm{kg}$. The subject was continually monitored by the attending physician for signs and symptoms of $N$-acetylcysteine reaction. Diphenhydramine hydrochloride (Benadryl; Parke-Davis, Morris Plains, NJ) was administered by intravenous injection as needed to lessen side-effects during $\mathrm{N}$-acetylcysteine infusion. All but one subject received an initial 5-mg bolus $15 \mathrm{~min}$ after the $\mathrm{N}$-acetylcysteine infusion began; additional 5-mg injections were administered as required to minimize discomfort. Cumulative diphenhydramine doses ranged from $0-25 \mathrm{mg}$ and averaged $13.5 \mathrm{mg} \pm 7.5 \mathrm{SD}$. For control trials, the infusion did not contain $\mathrm{N}$-acetylcysteine but diphenhydramine was administered using the same dosage and schedule as in treatment trials. To insure complete recovery between trials, treatment and control trials were performed 5-7 d apart. The order of control and treated trials was varied among subjects.

\section{Muscle preparation}

Isometric force development was measured during maximum voluntary contraction (MVC) of ankle dorsiflexors and during electrically evoked contractions of the tibialis anterior muscle as described previously (11). In brief, each subject was seated with knees and ankles at 90 degree angles and each foot resting on a rigid foot plate. Straps were placed around the subject's waist, thighs, and knees and a towel roll was placed between the knees in order to keep the subject in a stable position. Each foot support was equipped with a strain gauge to measure the forces developed by ankle dorsiflexor muscle groups including tibialis anterior. The strain gauge was calibrated before experiments by applying graded loads of known weight. The foot was strapped onto the support plate using a canvas strap positioned across the metatarsal heads. The strap was attached to the strain gauge and was tightened to a resting force of 45 Newtons (N). Force was recorded on a strip chart recorder.

We located the motor point for tibialis anterior by administering repetitive, transcutaneous stimuli (0.2-ms square wave pulses, 10-12 $\mathrm{mA}, 1 \mathrm{~Hz}$ ) using a constant-current stimulator and a bipolar probe (interelectrode distance $=7 \mathrm{~cm}$ ). Strength of contraction was documented by recording twitch force. The motor point was defined as the stimulation site that elicited the strongest twitch contraction. Carboncoated rubber electrodes $\left(5 \mathrm{~cm}^{2}\right)$ were positioned over the motor point (cathode) and $7 \mathrm{~cm}$ distally (anode). Maximum tolerable current was determined using repetitive tetanic stimuli. Current was increased until a 650 -ms stimulus train at $40 \mathrm{~Hz}$ was reported by the subject to be painful (i.e., pain threshold). The current then was decreased (typically by $2-3 \mathrm{~mA}$ ) to a level just below the pain threshold.

\section{Experimental protocols}

Two protocols were used to produce fatigue. Each was patterned after protocols developed previously (11) and included rigorous standardization of the number and timing of muscle stimulations. Submaximal tetanic contractions were stimulated repetitively using either near-minimal (low) or near-maximal (high) stimulation frequencies. Despite using different stimulus frequencies, each protocol was designed to produce a progressive $60-65 \%$ decrease in force output. This goal required that low-frequency stimulations (protocol 1) be administered using a higher duty cycle and a longer period of fatiguing contractions than were used during high-frequency stimulations (protocol 2). Acute recovery from fatigue also was measured, as were strength and contractile properties of unfatigued muscles as detailed below:

Protocol 1. Muscle strength, force-frequency characteristics, fatigue during low-frequency stimulation, and recovery. Before fatigue of each muscle, MVC was measured in triplicate and the force-frequency relationship was determined by stimulating tibialis anterior at frequencies of 1 (twitch), 10, 20, 40,80,100, and $120 \mathrm{~Hz}$ ( 5 contractions/frequency; $850 \mathrm{~ms}$ train duration). Fatigue was measured over $30 \mathrm{~min}$ using 850-ms trains of $10 \mathrm{~Hz}$ stimuli delivered each second (duty cycle 0.85 ). Acute recovery from fatigue was measured 10, 20, 30, 60, 90, $120,180,240$, and $300 \mathrm{~s}$ after fatiguing contractions were completed. At each time point, three 850 -ms trains of $10 \mathrm{~Hz}$ stimuli were delivered at $1 \mathrm{~s}$ intervals. Finally, the force-frequency relationship was remeasured in the muscle after partial recovery from fatigue.

Protocol 2. Fatigue during high-frequency stimulation and recovery. MVC was measured in triplicate. Fatigue then was produced using a protocol described in detail previously (11). In brief, trains of highfrequency $(40 \mathrm{~Hz}$ ) stimuli were delivered each second (duty cycle 0.65 ) for $180 \mathrm{~s}$. Recovery from fatigue was measured 10, 20, 30, 60, 90, 120, 180,240 , and $300 \mathrm{~s}$ after fatiguing contractions ended. At each time point, three 650 -ms trains of $40 \mathrm{~Hz}$ stimuli were delivered at $1 \mathrm{~s}$ intervals.

\section{Data analysis}

Data from individual muscles were accepted for analysis if $(a)$ electrically evoked contractions were reproducible before fatigue, (b) $40 \mathrm{~Hz}$ tetanic contractions developed $\geq 100 \mathrm{~N},(c)$ force fell monotonically during fatigue without evidence of concurrent activity by agonist or antagonist muscle groups, and $(d)$ baseline force remained stable throughout the fatigue trial. Experiments on 26 muscles produced 22 data sets of two trials each that met the above criteria. In data sets from four muscles, one or both trials did not meet these criteria (e.g., Fig. 1); these data sets were excluded from subsequent analyses. Fatigue and recovery data were normalized by expressing the forces developed during each trial as a percentage of the initial force developed at time 0 . Forces developed during the three MVC maneuvers were averaged to obtain a single value for each experiment. Average force-frequency relationships were constructed by expressing the forces developed at stimulus frequencies of $1,10,20,40$, and $120 \mathrm{~Hz}$ as percentages of maximal tetanic force developed at $80 \mathrm{~Hz}$. Data were analyzed as time- 


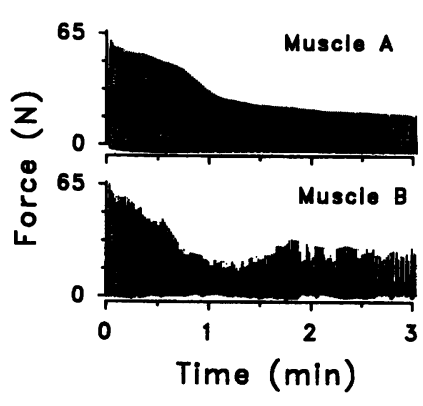

and stimulus-matched, paired observations, comparing treated vs. control conditions within each muscle.

\section{Statistics}

Differences between trials in stimulus current and force production (Table I) were evaluated using Student's $t$ test for paired data (18). Changes in force as a function of time (Figs. 2, 4, 5) or stimulus frequency (Fig. 6) were tested for a main effect of treatment using two-way analyses of variance for repeated measures (19); post hoc tests for differences at individual time points employed the linear contrast technique (19). Two-tailed $P$ values $<0.05$ were considered significant except in posthoc analyses where Bonferonni corrections were applied. Averaged data are reported $\pm \mathrm{SE}$.

\section{Results}

\section{$N$-acetylcysteine effects on development and reversal of} fatigue

Low-frequency stimulation. During repetitive low-frequency stimulation (Fig. 2), force fell progressively over $30 \mathrm{~min}$. The rate of decline was biphasic, falling rapidly over the first 5$10 \mathrm{~min}$ and more slowly thereafter. $\mathrm{N}$-acetylcysteine inhibited fatigue under these conditions. Compared to control trials, the forces produced by treated muscles were significantly elevated after $3 \mathrm{~min}$ of activity and remained elevated throughout the remainder of the trial. Fig. 3 depicts the time-averaged responses of individual muscles. Over the entire protocol, $\mathrm{N}$-acetylcysteine treatment enhanced average force output in six of eight muscles studied. Data in Fig. 4 depict acute recovery from fatigue produced using low-frequency stimulation. During the first $3 \mathrm{~min}$, force developed at $10 \mathrm{~Hz}$ increased from 39 to $66 \%$ of the

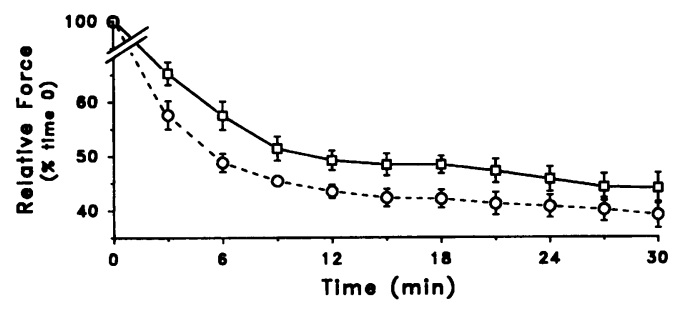

Figure 2. $\mathrm{N}$-acetylcysteine inhibits low-frequency fatigue (protocol 1). Values are relative forces ( \pm SE) developed by muscles during fatiguing tetanic ( $10 \mathrm{~Hz}$ ) contractions after infusion of $N$-acetylcysteine $150 \mathrm{mg} /$ $\mathrm{kg}$ (open squares) or diluent (open circles); repeated measures ANOVA confirmed that force development fell over time $(P<0.0001)$ and was greater after treatment $(P<0.0001)$; there was no interaction of time and treatment effects on force $(P<0.80)$.

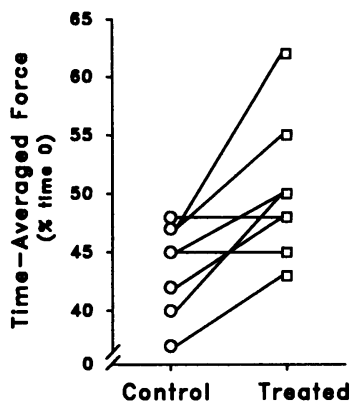

Figure 3. Sensitivity of individual muscles to $\mathrm{N}$-acetylcysteine (protocol 1). Values depict time-averaged forces developed by individual muscles from 3 through 30 min of fatiguing $10 \mathrm{~Hz}$ contractions; control (open square) and treated trials (open circle) of each muscle are linked by a solid line; $N$-acetylcysteine increased force production in six of eight muscles studied.

prefatigue level. This increase was unaffected by $N$-acetylcysteine.

High-frequency stimulation. Fatigue induced using highfrequency stimulation (Fig. 5) resulted in a rapid, biphasic decrease in force to $34 \%$ of prefatigue values. Acute recovery was more complete than in low-frequency trials; average force increased to $84 \%$ of the prefatigue level within $3 \mathrm{~min}$. Both fatigue at $40 \mathrm{~Hz}$ and recovery were insensitive to $N$-acetylcysteine; forces were not different between treated and control trials at any time point.

\section{$N$-acetylcysteine effects on unfatigued muscle}

$\mathrm{N}$-acetylcysteine treatment did not influence volitional performance of MVC maneuvers (Table I). Maximal forces developed using ankle dorsiflexor muscle groups ranged from 266 to $540 \mathrm{~N}$ and did not differ after treatment in either protocol. The response of tibialis anterior to electrical activation also was unaltered by $N$-acetylcysteine. Within each protocol, stimuli applied to the motor point during treated and control trials were matched for current intensity and produced equivalent contractile responses (Table I). Combining data from control and treated trials, maximal tetanic $(80 \mathrm{~Hz})$ stimulation of tibialis anterior generated forces that averaged $34 \%$ of MVC $( \pm 2)$; forces developed at the beginning of fatigue protocols averaged $11 \% \pm 1(10 \mathrm{~Hz})$ and $38 \% \pm 2(40 \mathrm{~Hz})$ of MVC. As shown in Fig. 6, force development and stimulation frequency exhibited a typical sigmoidal relationship that was shifted down and to the right following fatigue produced using low-frequency stimulation. $\mathrm{N}$-acetylcysteine had no effect on this relationship in either condition.

\section{Systemic reactions to $N$-acetylcysteine}

We observed the signs and symptoms listed in Table II in our subjects. The average time to onset of side effects after initiation of the $N$-acetylcysteine infusion was $\sim 30$ min with a range of $15 \mathrm{~s}$ (infusion site burning and erythema) to $65 \mathrm{~min}$ (emesis). Data in Fig. 7 show that the incidence of side effects increased progressively during the first 45-50 min of $N$-acetylcysteine

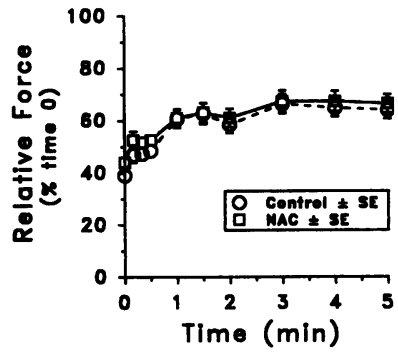

Figure 4. Acute recovery from low-frequency fatigue (protocol 1). Values depict relative forces $( \pm \mathrm{SE})$ developed during periodic tetanic $(10 \mathrm{~Hz})$ contractions immediately following low-frequency fatigue; force increased modestly from 39 to $66 \%$ and was uninfluenced by $\mathrm{N}$-acetylcysteine pretreatment. 


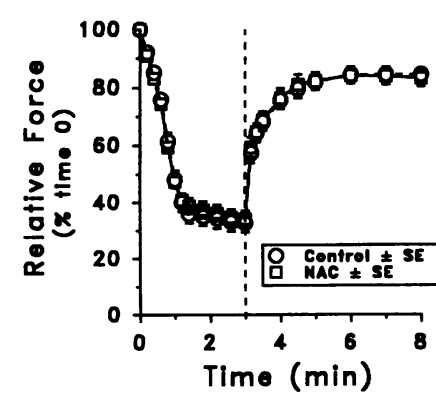

Figure 5. Development and acute reversal of high-frequency fatigue (protocol 2). Values depict relative forces $( \pm \mathrm{SE})$ developed during repetitive, nearmaximal tetanic $(40 \mathrm{~Hz})$ contractions timed to produce fatigue $(0-3 \mathrm{~min})$ or to allow acute reversal of fatigue $(3-8$ $\min$ ) following infusion of $N$ acetylcysteine (open squares) or diluent (open circles); a vertical dashed line at $3 \mathrm{~min}$ delineates the end of fatiguing contractions; treatment had no effect on development or reversal of fatigue using 40 $\mathrm{Hz}$ stimulation.

infusion, remained elevated for $\sim 20 \mathrm{~min}$, then progressively declined. Side effects were dose dependent. Two subjects, during separate experimental protocols, received doses of 50,100, and $150 \mathrm{mg} / \mathrm{kg}$ of $\mathrm{N}$-acetylcysteine with no change in the type or pattern of side effects but with a progressive increase in severity. Diphenhydramine blunted but did not completely treat side effects. Central nervous system (CNS) symptoms appeared to be least responsive to diphenhydramine while pulmonary, gastrointestinal, and conjunctival symptoms rapidly abated. Five subjects reported symptoms that are not presented in the table or figure because these occurred after completion of the experimental protocols. These subjects reported gait disturbances, primarily difficulty in maintaining balance with walking. Stable posturography was tested in two individuals and was normal.

\section{Discussion}

These experiments are the first to demonstrate that antioxidant pretreatment can inhibit fatigue of human skeletal muscle. Intravenous infusion of $\mathrm{N}$-acetylcysteine $150 \mathrm{mg} / \mathrm{kg}$ inhibited development of fatigue in tibialis anterior during repetitive, lowfrequency electrical stimulation. $N$-acetylcysteine did not alter other aspects of muscle function, i.e., fatigue during high-frequency stimulation, acute recovery from fatigue, strength of unfatigued muscle, or contractile properties. These findings indicate that oxidative stress contributes to loss of function in human muscle fatigue and that peripheral fatigue can be selec-

Table I. Forces Developed by Unfatigued Muscles (N)

\begin{tabular}{lccc}
\hline & Protocol & Control & Treated \\
\hline MVC & 1 & $384 \pm 84$ & $391 \pm 24$ \\
& 2 & $403 \pm 19$ & $396 \pm 20$ \\
Maximal tetanus* & 1 & $131 \pm 7$ & $129 \pm 8$ \\
& 2 & - & - \\
Fatigue protocol at time $0^{\ddagger}$ & 1 & $46 \pm 7$ & $46 \pm 6$ \\
& 2 & $144 \pm 6$ & $148 \pm 6$ \\
\hline
\end{tabular}

All values are means \pm SE; $n=8$ in protocol $1, n=14$ in protocol 2; MVC, maximal voluntary contraction; * forces developed during maximal tetanic stimulation at $80 \mathrm{~Hz}$ (protocol 1 only); ${ }^{\ddagger}$ forces developed during submaximal tetanic stimulation using $10 \mathrm{~Hz}$ (protocol 1) or 40 $\mathrm{Hz}$ (protocol 2) at time $\mathbf{0}$ of fatigue trials.

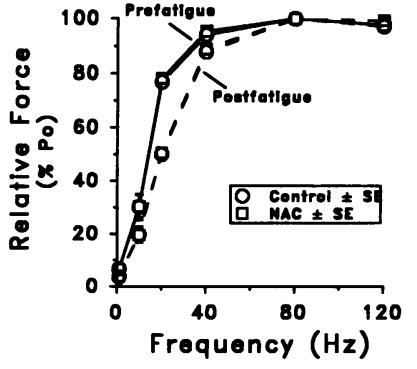

Figure 6. Force-frequency characteristics before and after low-frequency fatigue (protocol 1). Curves depict relative forces ( shown as means $\pm \mathrm{SE} ; n=8$ ) developed by tibialis anterior stimulated at 1, 10, 20,40,80 (maximal tetanus), and $120 \mathrm{~Hz}$; measurements were made before fatiguing contractions at $10 \mathrm{~Hz}$ (Prefatigue, solid lines) and after (Postfatigue, dashed lines); forces developed after $N$-acetylcysteine treatment (open squares) were not different from untreated control (open circles) at any frequency pre- or postfatigue; differences between prefatigue and postfatigue relationships are highly significant $(P<0.001)$.

tively inhibited by pretreating individuals with an exogenous antioxidant.

Electrically stimulated fatigue. In this study, we produced peripheral fatigue in human tibialis anterior using a previously developed technique (11) that provides tight control over experimental conditions. The muscle is activated electrically using repetitive trains of tetanic stimuli and isometric force is measured. Fatigue is characterized by a monotonic fall in force that is proportional to duty cycle, is highly reproducible for individual muscles, and reverses when stimulation ceases (11).

Protocols 1 and 2 produced comparable degrees of fatigue (declines in relative force) using either low or high stimulus frequencies, interventions that are thought to produce fatigue by different cellular mechanisms. Fatigue produced using low stimulus frequencies closely approximates the biochemical changes that occur during volitional exercise: progressive decreases in developed force are associated with lactic acidosis and accumulation of inorganic phosphate which contribute directly to mechanical failure by inhibiting actin-myosin interaction (12). Fatigue induced using low stimulus frequencies preferentially depresses tetanic force development at low frequencies with relatively less effect on force output at high frequencies (20) as observed in this study. Recovery from this form of fatigue is a slow process that typically requires hours to days for complete reversal, another characteristic that mimics

Table II. Signs and Symptoms of $\mathrm{N}$-acetylcysteine Administration

\begin{tabular}{lrc}
\hline & \multicolumn{2}{c}{ No. of occurrences } \\
\cline { 2 - 3 } \multicolumn{1}{c}{ Signs and symptoms } & Treated & Control \\
\hline Conjunctival irritation & 11 & 0 \\
Dysphoria & 8 & 0 \\
Sleepiness & 7 & 8 \\
Cough & 7 & 0 \\
Lightheadedness & 6 & 0 \\
Palmar erythema/sweating & 6 & 0 \\
Facial erythema & 5 & 0 \\
Dyspepsia & 5 & 0 \\
Nausea & 4 & 0 \\
Pruritus & 3 & 0 \\
Metallic taste & 3 & 1
\end{tabular}

Values are combined occurrences in 13 experiments on 10 subjects in protocols 1 and 2. 


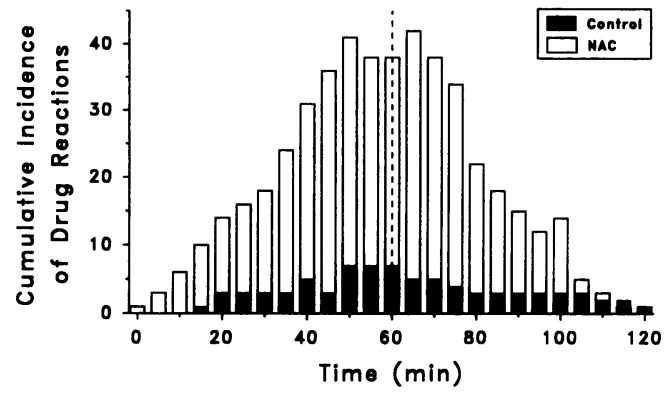

Figure 7. Time course of reactions to $N$-acetylcysteine infusion. Values depict combined signs and symptoms reported by all subjects during and immediately following infusion of $\mathrm{N}$-acetylcysteine (open bars) or diluent (closed bars); a vertical dashed line at 60 min marks the end of infusion; diphenhydramine was administered to each subject using an individualized dosage and schedule that was identical between treated and control trials and was responsible for reactions observed during control trials.

volitional fatigue (20). Our measurements of acute recovery reflect only the initial phase of this extended process.

Fatigue produced by high-frequency stimulation causes loss of contractile function by a different mechanism that is characterized by failure of t-tubule depolarization, decreased $\mathrm{Ca}^{2+}$ release from the sarcoplasmic reticulum, and disruption of excitation-contraction coupling (12). The ionic imbalance in the ttubules undergoes rapid reversal once muscle stimulation ceases contributing to the rapid recovery of mechanical function that we observed. The physiological relevance of this form of fatigue remains controversial (12).

Antioxidants and muscle fatigue. Fatigue induced using low stimulus frequencies was inhibited by systemically pretreating subjects with $N$-acetylcysteine. Our findings are similar to data obtained from anesthetized rabbits by Shindoh and colleagues (15) who evaluated $\mathrm{N}$-acetylcysteine effects on diaphragm strips in situ. During a 20-min protocol of low-frequency stimulation via the phrenic nerve, isometric force declined more slowly in animals that had been pretreated by intravenous infusion of $N$-acetylcysteine $150 \mathrm{mg} / \mathrm{kg}$ than in untreated animals. As in our study, Shindoh et al. observed that acute recovery from fatigue and the force-frequency relationship were unaffected by this dose of $\mathrm{N}$-acetylcysteine. Systemic pretreatment with other antioxidant compounds also has been shown to blunt peripheral fatigue. Barclay and Hansel (21) reported that intravenous administration of either deferoxamine, dimethyl sulfoxide, or allopurinol can slow the fall of isometric force during repetitive, low-frequency stimulation of canine gastrocnemius in situ. Antioxidant administration is not uniformly beneficial, however. Fatigue induced using high stimulus frequencies has not been influenced by antioxidant pretreatment either in the present human studies or in studies of isolated rodent muscle $(16,17)$. Long-term supplementation of antioxidant nutrients has not improved human exercise endurance (6-9) nor has systemic administration of oxygen radical scavengers influenced electrically stimulated fatigue of canine limb muscle in situ (22). Negative results suggest that antioxidant efficacy may depend critically on the agent administered, the dosage, and the pattern of muscular activity used to induce fatigue.

Little presently is known about the mechanism(s) by which $\mathrm{N}$-acetylcysteine and other antioxidants inhibit fatigue. In vitro studies of curarized, directly stimulated muscle have shown that
$N$-acetylcysteine $(13,14)$ and other antioxidants $(16,17)$ can inhibit fatigue via direct effects on the myocyte. Presumably, exogenous antioxidants remove reactive oxidants (e.g., free radicals, hydroperoxides) that are produced during strenuous muscular contractions $(1,17,23,24)$ thereby limiting development of oxidative stress and maintaining redox-sensitive functions within the cell closer to prefatigue levels.

In theory, direct effects of $\mathrm{N}$-acetylcysteine on fatiguing muscle may have been enhanced by indirect effects of the drug on muscle perfusion. Pharmacologic vasodilators can inhibit peripheral fatigue (25) and $\mathrm{N}$-acetylcysteine has vasodilatory activity, potentiating the effects of endogenous nitric oxide (26) and increasing organ blood flow (27) under certain conditions. $\mathrm{N}$-acetylcysteine did not alter blood pressure or heart rate in our subjects. Blood flow to the working muscle was not measured, however, and the effect of $N$-acetylcysteine on exercise hyperemia or control of muscle blood flow has not been investigated. Vasodilation therefore cannot be ruled out as a potential mechanism of action.

$N$-acetylcysteine pharmacology. $N$-acetylcysteine (MW 163 ) is a sulfhydryl-containing antioxidant used clinically as an acute antidote to acetaminophen overdose and as a pulmonary mucolytic agent (10). $N$-acetylcysteine reacts nonspecifically with a wide variety of reactive oxidants, including free radicals and reactive oxygen intermediates. $N$-acetylcysteine has not been shown to cross the sarcolemma but can directly inactivate electrophils in the extracellular compartment via either reduction or conjugation reactions. $\mathrm{N}$-acetylcysteine may indirectly exert intracellular effects by serving as a source of reduced cysteine and thereby supporting glutathione biosynthesis. This is the predominant mechanism of action in acetaminophen-induced hepatotoxicity (28). The mechanism of $N$-acetylcysteine action in skeletal muscle is poorly characterized and the relative importance of direct vs. indirect antioxidant activities has not been examined.

Systemic pharmacokinetics of $\mathrm{N}$-acetylcysteine have been described in humans by Olsson and colleagues (29). Intravenous infusion of $150 \mathrm{mg} / \mathrm{kg}$ over $15 \mathrm{~min}$ achieves a mean plasma concentration of $3.4 \mu \mathrm{mol} /$ liter $( \pm 0.8 \mathrm{SE})$ that is maximal at end-infusion. The plasma concentration of total $\mathrm{N}$-acetylcysteine declines with a half-life of $\sim 5.5 \mathrm{~h}$ whereas reduced $\mathrm{N}$-acetylcysteine disappears more rapidly (half-life $1.97 \mathrm{~h}$ ) due primarily due to formation of mixed disulfides with plasma proteins. The volumes of distribution for total and reduced $N$ acetylcysteine are 0.47 and $0.59 \mathrm{liter} / \mathrm{kg}$, respectively.

In the absence of acetaminophen toxicity, the side effects of intravenous $\mathrm{N}$-acetylcysteine administration are poorly understood. This is the first prospective analysis in normal subjects. Systemic reactions to $\mathrm{N}$-acetylcysteine were anaphylactoid and dose dependent as reported retrospectively after acute treatment of paracetamol overdose $(30,31)$. The incidence and intensity of the reactions that we observed were unexpectedly high. All subjects experienced one or more side effects. In early trials (two preliminary; one formal), three subjects chose to receive $N$-acetylcysteine without diphenhydramine. Each developed prominent reactions that were decidedly unpleasant and temporarily debilitating. Diphenhydramine administration therefore was required in all subsequent studies and proved effective in blunting peripheral side effects. Diphenhydramine was relatively ineffective against CNS reactions that altered motor control, an effect of $\mathrm{N}$-acetylcysteine that previously had not been reported and that persisted longer than other symptoms 
in our subjects. In contrast to the relatively benign side effects observed in our experiments, $\mathrm{N}$-acetylcysteine overdose can have drastic consequences. Toxic reactions may include severe bronchospasm, angioedema, and hypotension and can be fatal in some individuals as recently reviewed by Flanagan and Meridith (10).

Clinical relevance. Premature fatigue of limb muscles impairs patient mobility in a variety of neurologic disorders, cardiovascular diseases, and primary myopathies. Fatigue of the respiratory muscles may have more serious consequences, resulting in ventilatory insufficiency and mechanical ventilation. Accordingly, pharmacologic compounds that might inhibit fatigue of limb and respiratory skeletal muscles have been the focus of extensive research but no effective intervention has yet been identified $(6,8,32,33)$.

In this study, $N$-acetylcysteine increased the forces sustained during peripheral fatigue by $\sim 15 \%$. An increment of this magnitude could be beneficial (imagine, for example, the potential advantage to an Olympic athlete) but is not necessarily the upper limit of antioxidant efficacy. Shindoh and colleagues (15) found that the forces sustained during fatiguing contractions by rabbit diaphragm in situ were threefold greater than in the present study $(\sim 50 \%)$, suggesting that $N$-acetylcysteine effects may vary among individual muscles or among species. It may be possible to enhance the action of $N$-acetylcysteine by using a different mode of administration. For intravenous infusion, an equilibration period before fatigue trials might allow greater $\mathrm{N}$-acetylcysteine uptake from the vascular compartment and thereby enhance efficacy. Oral administration of $\mathrm{N}$-acetylcysteine has been shown to increase glutathione levels in plasma, hepatocytes, and bronchoalveolar lavage fluid of humans (28) and also might have beneficial effects on myocytes. Finally, other antioxidant compounds may prove to be more effective against fatigue than $\mathrm{N}$-acetylcysteine. Supplementation of antioxidant nutrients (e.g., selenium, vitamin c, alpha tocopherol) generally has not increased human endurance during exercise (6-9) but other antioxidants are approved for human administration, e.g., allopurinol, native and esterified glutathione, superoxide dismutase, and lazaroids (34), and may prove beneficial. To date, however, none has been tested for effects on human muscle function or exercise performance.

Therapeutic applications of $N$-acetylcysteine will be limited by side effects of the drug. Volitional performance of simple MVC maneuvers was unaffected by $N$-acetylcysteine administration; however, losses in CNS function (e.g., dynamic balance, motor control) and the distraction of systemic reactions (nausea, dysphoria, etc.) would have seriously complicated the performance of whole-body exercise (e.g., cycling, running). It is likely that the side effects of $N$-acetylcysteine would outweigh direct effects on muscle endurance, causing a paradoxical decrease in exercise performance. Continued research may identify an effective antioxidant with lesser side effects but our experience suggests that $\mathrm{N}$-acetylcysteine is more useful as an experimental tool than as an ergogenic aid.

Summary and conclusions. This study demonstrates that $N$ acetylcysteine inhibits fatigue of human tibialis anterior during repetitive, low-frequency electrical stimulation. $N$-acetylcysteine did not alter other aspects of muscle function, i.e., fatigue during high-frequency stimulation, acute recovery from fatigue, strength of unfatigued muscle, or contractile properties. The importance of these findings is twofold. First, our data indicate that oxidative stress may play a causal role in human muscle fatigue. Previous measurements of glutathione oxidation and lipid peroxidation therefore may reflect a cause of fatigue in exercising humans and not simply a deleterious effect. Second, positive effects of antioxidant pretreatment have important practical implications for the fields of exercise physiology, restorative neurology, and sports medicine wherein antioxidant administration may provide a novel intervention for experimental or therapeutic use.

\section{Acknowledgments}

The authors thank Apothecon for the generous gift of Mucomyst Intravenous ( $N$-acetylcysteine), our volunteer subjects for their cooperation, Drs. Owen B. Wilson and Charles C. Miller III for statistical advice, Tina Martinez-Barrett for secretarial assistance, and Melanie M. Moody for assistance with figures.

This study was supported by National Institutes of Health grant HL45721 (M. Reid), US Public Health Service grant RR-05425 (A. Leis), and the Vivian L. Smith Foundation for Restorative Neurology.

\section{References}

1. Jackson, M. J., R. H. T. Edwards, and M. C. R. Symons. 1985. Electron spin-resonance studies of intact mammalian skeletal muscle. Biochim. Biophys. Acta. 847:185-190.

2. Ji, L. L., A. Katz, R. Fu, M. Griffiths, and M. Spencer. 1993. Blood glutathione status during exercise: effect of carbohydrate supplementation. $J$. Appl. Physiol. 74:788-792.

3. Gohil, K., C. Viguie, W. C. Stanley, G. A. Brooks, and L. Packer. 1988. Blood glutathione oxidation during human exercise. J. Appl. Physiol. 64:115119.

4. Kanter, M. M., L. A. Nolte, and J. O. Holloszy. 1993. Effects of an antioxidant vitamin mixture on lipid peroxidation at rest and postexercise. J. Appl. Physiol. 74:965-969.

5. Sumida, S., K. Tanaka, H. Kitao, and F. Nakamodo. 1989. Exercise-induced lipid peroxidation and leakage of enzymes before and after vitamin E supplementation. Int. J. Biochem. 21:835-838.

6. Jackson, M. J., and R. H. Edwards. 1990. Free radicals and trials of antioxidant therapy in muscle diseases. Adv. Exp. Med. Biol. 264:485-491.

7. Witt, E. H., A. Z. Reznick, C. A. Viguie, P. Starke-Reed, and L. Packer. 1992. Exercise, oxidative damage, and effects of antioxidant manipulation. $J$. Nutr. 122:766-773.

8. Goldfarb, A. H. 1993. Antioxidants: role of supplementation to prevent exercise-induced oxidative stress. Med. Sci. Sports Exercise. 25:232-236.

9. Weight, L. M., K. H. Myburgh, and T. D. Noakes. 1988. Vitamin and mineral supplementation: effect on the running performance of trained athletes. Am. J. Clin. Nutr. 47:192-195.

10. Flanagan, R. J., and T. J. Meredith. 1991. Use of N-acetylcysteine in clinical toxicology. Am. J. Med. 91:131S-139S.

11. Reid, M. B., G. J. Grubweiser, D. S. Stokic, S. M. Koch, and A. A. Leis. 1993. Development and reversal of fatigue in human tibialis anterior. Muscle Nerve. 16:1239-1245.

12. Westerblad, H., J. A. Lee, J. Lannergren, and D. G. Allen. 1991. Cellular mechanisms of fatigue in skeletal muscle. Am. J. Physiol. 261:C195-C209.

13. Diaz, P. T., and T. L. Clanton. 1992. Fatigue-sparing effects of acetylcysteine on the diaphragm are temperature-dependent. Am. Rev. Respir. Dis. 145:A150. (Abstr.)

14. Khawli, F. A., and M. B. Reid. 1994. N-acetylcysteine depresses contractility and inhibits fatigue of diaphragm in vitro. J. Appl. Physiol. 77:317-324.

15. Shindoh, C., A. Dimarco, A. Thomas, P. Manubray, and G. Supinski. 1990. Effect of $N$-acetylcysteine on diaphragm fatigue. J. Appl. Physiol. 68:21072113.

16. Supinski, G., D. Nethery, and A. Dimarco. 1991. Effect of a free radical scavenger on lipid peroxidation in the fatiguing diaphragm. Am. Rev. Respir. Dis. 143:A560. (Abstr.)

17. Reid, M. B., K. E. Haack, K. M. Franchek, P. A. Valberg, L. Kobzik, and M. S. West. 1992. Reactive oxygen in skeletal muscle: I. Intracellular oxidant kinetics and fatigue in vitro. J. Appl. Physiol. 73:1797-1804.

18. Zar, J. H. 1974. Biostatistical Analysis. Prentice-Hall, Inc., Englewood Cliffs, NJ. 620 pp.

19. SAS Institute, Inc. 1988. The GLM procedure. In SAS/STAT User's Guide, Release 6.03 Edition. SAS Institute, Inc., Cary, NC.

20. Edwards, R. H. T., D. K. Hill, D. A. Jones, and P. A. Merton. 1977. 
Fatigue of long duration in human skeletal muscle after exercise. J. Physiol. 272:769-778.

21. Barclay, J. K., and M. Hansel. 1991. Free radicals may contribute to oxidative skeletal muscle fatigue. Can. J. Physiol. Pharmacol. 69:279-284.

22. Shrier, I., S. Hussain, and S. Magder. 1991. Failure of oxygen radical scavengers to modify fatigue in electrically stimulated muscle. Can. J. Physiol. Pharmacol. 69:1470-1475.

23. Reid, M. B., T. Shoji, M. R. Moody, and M. L. Entman. 1992. Reactive oxygen in skeletal muscle: II. Extracellular release of free radicals. J. Appl. Physiol. 73:1805-1809.

24. Diaz, P. T., Z.-W. She, W. B. Davis, and T. L. Clanton. 1993. Hydroxylation of salicylate by the in vitro diaphragm: evidence for hydroxyl radical production during fatigue. J. Appl. Physiol. 75:540-545.

25. Supinski, G. S., A. F. DiMarco, J. Gonzales, and M. D. Altose. 1990. Effect of norepinephrine on diaphragm contractility and blood flow. J. Appl. Physiol. 69:2019-2028.

26. Simon, D. I., J. S. Stamler, O. Jaraki, J. F. Keaney, J. A. Osborne, S. A. Francis, D. J. Stengel, and J. Loscalzo. 1993. Antiplatelet properties of protein $\mathrm{S}$-nitrosothiols derived from nitric oxide and endothelium-derived relaxing factor. Arterioscler. Thromb. 13:791-799.
27. Boesgaard, S., H. K. Iversen, H. Wroblewski, H. E. Poulsen, H. Frandsen, J. Kastrup, and J. Aldershvile. 1994. Altered peripheral vasodilator profile of nitroglycerine during long-term infusion of $\mathrm{N}$-acetylcysteine. J. Am. Coll. Cardiol. 23:163-169.

28. Ruffmann, R., and A. Wendel. 1991. GSH rescue by $N$-acetylcysteine. Klin. Wochenschr. 69:857-862.

29. Olsson, B., M. Johansson, J. Gabrielsson, and P. Bolme. 1988. Pharmacokinetics and bioavailability of reduced and oxidized $\mathrm{N}$-acetylcysteine. Eur. J. Clin. Pharmacol. 34:77-82.

30. Mant, T. G. K., J. H. Tempowski, G. N. Volans, and J. C. C. Talbot. 1984. Adverse reactions to acetylcysteine and effects of overdose. Br. Med. J. 289:217-222.

31. Dawson, A. H., D. A. Henry, and J. McEwen. 1984. Adverse reactions to $\mathrm{N}$-acetylcysteine during treatment for paracetamol poisoning. Med. J. Aust. 150:329-331.

32. Esau, S. A., and M. B. Reid. 1992. Pharmacologic enhancement of respiratory muscle function. Semin. Respir. Med. 13:33-43.

33. Williams, M. H. 1992. Ergogenic and ergolytic substances. Med. Sci. Sports Exercise. 24:S344-S348.

34. Halliwell, B. 1991. Drug antioxidant effects. A basis for drug selection? Drugs. 42:569-605. 